\title{
Follicular bronchiolitis in primary ciliary dyskinesia
}

\author{
Prashanth M Thalanayar ${ }^{1}$, Fernando \\ Holguin ${ }^{2}$
}

1. Dept. of Internal medicine, University of Pittsburgh Medical Center, McKeesport, PA, USA

2. Asthma Institute, University of Pittsburgh Medical Center, Pittsburgh, PA, USA

\section{CASE REPORT}

Please cite this paper as: Thalanayar PM, Holguin F. Follicular bronchiolitis in primary ciliary dyskinesia. AMJ 2014, 7, 7, 294-297. http://doi.org/10.21767/AMJ.2014.2102

Corresponding Author:

Prashanth M Thalanayar

University of Pittsburgh Medical Center McKeesport

1500 Fifth Avenue

McKeesport PA 15132

USA

Email: thalanayarp@upmc.edu

\section{ABSTRACT}

Ciliary dysfunction in primary ciliary dyskinesia (PCD) may be associated with bronchiolitis. Diffuse bronchiolitis has been reported in a subset of PCD patients who have Kartagener's syndrome in Japan. We report a case of follicular bronchiolitis (FB) in a case of PCD presenting with recurrent episodes of cough, dyspnea, and bronchiectasis. This may motivate researchers to study rarer variants and presentations in PCD.

\section{Key Words}

Follicular bronchiolitis, Primary ciliary dyskinesia, Macrolides

\section{Implications for Practice:}

\section{What is known about this subject?}

Primary ciliary dyskinesia is rarely associated with bronchiolitis and the histopathology has been noted to be diffuse bronchiolitis in nature.

\section{What is the key finding in this case study?}

Follicular bronchiolitis in association with PCD has not been reported before. However, other subtypes of bronchiolitis are present in the literature.

\section{What are the implications for future practice?}

In patients with bronchiectasis of unknown aetiology, the presence of follicular bronchiolitis, while not specific, may be associated with PCD. In this clinical scenario, clinicians might consider a diagnostic work up for PCD. This case report may urge clinician scientists to attempt to identify histological patterns of bronchiolitis in PCD cohorts and study the distribution in the population so as to shed more light on this newly reported association.

\section{Background}

Primary ciliary dyskinesia (PCD) is a known congenital disorder manifesting in infancy or childhood in most cases. With regard to the respiratory apparatus, ciliary dysfunction results in recurrent sinusitis, pneumonia, and bronchiectasis usually before the individual has reached adulthood. ${ }^{1}$ Bronchiolitis has been reported in at least three case series of patients with primary ciliary dyskinesia, but the pathology in these cases has been suggestive of diffuse panbronchiolitis (DPB). ${ }^{2-4}$ We hereby report a unique case of $P C D$ with histopathological features not reported before in the medical literature.

\section{Case details}

We present a 27-year-old Caucasian woman with history of recurrent sinusitis, respiratory infections, and a diagnosis of asthma since childhood who presented to the emergency room (ER) in 2011 with complaints of cough and yellow sputum. Coarse breath sounds, rhonchi, and wheeze were found on examination. Chest $\mathrm{X}$-ray revealed left lower lobe pneumonia and she was treated with moxifloxacin as an outpatient. A similar subsequent hospitalisation was associated with left lower lobe opacities found on computed tomography (CT). Despite completing treatment, her cough persisted and she was managed conservatively on an outpatient basis.

She was readmitted to the hospital two months later with similar symptoms along with a fever and respiratory failure. This time CT chest revealed right middle lobe bronchiectasis along with alveolar "tree-in-bud" opacities in bilateral lower lobes (Figure 1). She received another 
course of moxifloxacin. However, during the whole of 2011, she continued to have multiple readmissions for shortness of breath and productive cough, and bronchiectasis involved a portion of the lower lobes, too (Figure 2). Bronchoscopy was undertaken and it revealed thick secretions emanating from the right lower lobe. Bronchoalveolar lavage fluid grew pseudomonas and viridans streptococci in culture. She was discharged home on ciprofloxacin after three days of intravenous (IV) piperacillin-tazobactam having noticed symptomatic and objective improvement.

She was seen later as an outpatient after interval improvement of the pneumonia. Repeat bronchoscopy was performed and pathology from trans-bronchial biopsies showed prominent B cell submucosal nodules with chronic inflammatory infiltration-consistent with follicular bronchiolitis (FB). No granulomas were reported (Figure 3). Investigation for cystic fibrosis, aspergillosis, hypogammaglobulinemia, immunodeficiency, rheumatoid arthritis, lupus, and connective tissue disorders were negative. Eventually, bronchoscopic biopsies from the carina were performed and they revealed the absence of ciliary beat. Electron microscopy of the respiratory epithelium revealed PCD with the lack of outer dynein arms.

Figure 1: CT scan depicting consolidation involving left lower lobe and bronchiectasis of right middle lobe

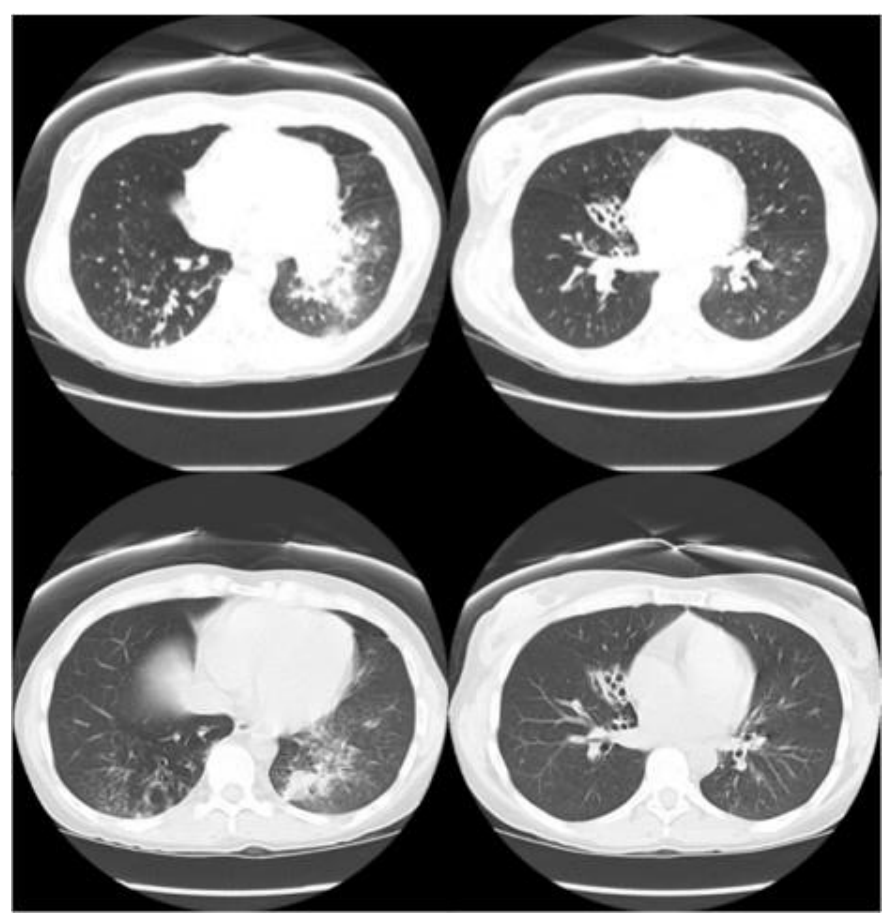

Figure 2: CT scan depicting consolidation involving right lower lobe and bronchiectasis of right middle lobe

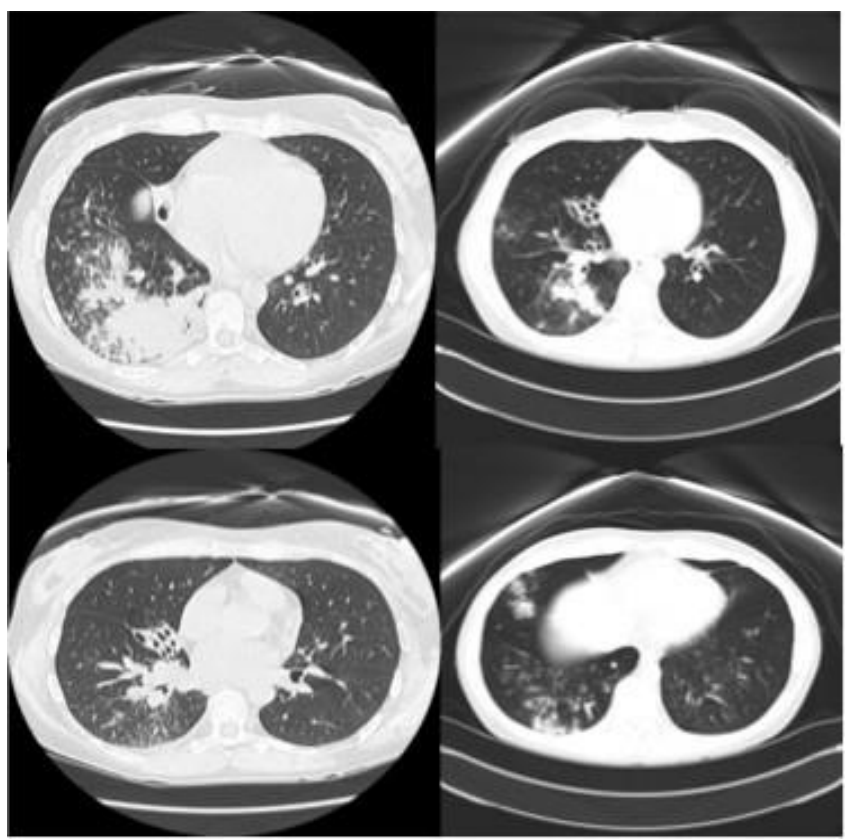

Figure 3: Histopathology depicting follicular bronchiolitis in airway epithelium

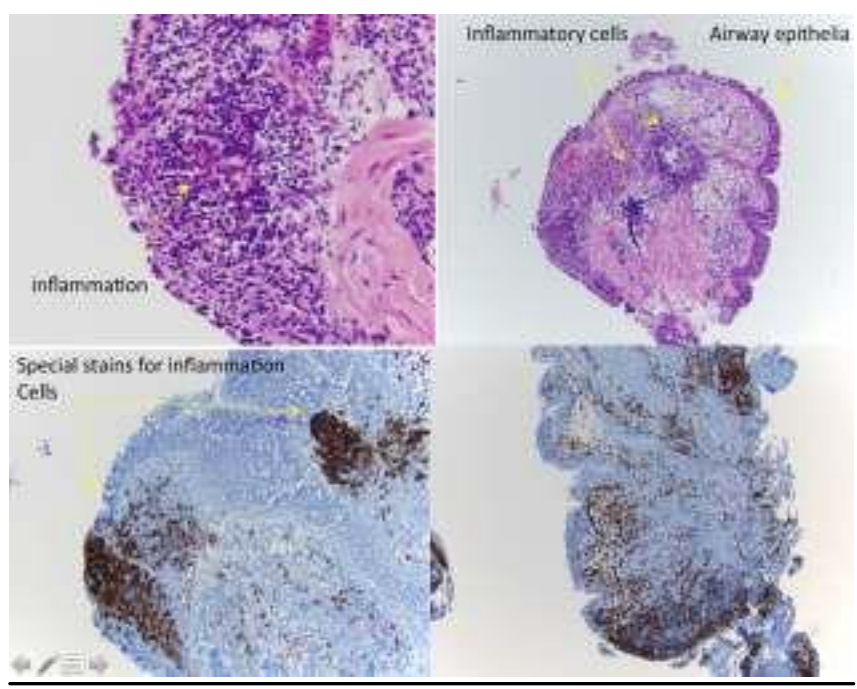

\section{Methods}

MEDLINE search was conducted using MeSH headings including follicular, bronchiolitis, primary ciliary dyskinesia, immotile cilia syndrome. The search revealed no previous reports of a follicular bronchiolitis histopathology seen with PCD.

\section{Discussion}

Stimulation of the bronchus-associated lymphoid tissue (BALT) resulting in a polyclonal hyperplasia of lymphoid follicles around the bronchioles is described as follicular bronchiolitis. Such reactive lymphoid follicles are situated between bronchioles and pulmonary arteries and may 
compress the bronchiolar lumen. Bronchiectasis may lead to or possibly result from compression by peri-bronchiolar infiltration. ${ }^{5}$ The distinguishing factor between lymphocytic interstitial pneumonia and follicular bronchiolitis is the diffuse infiltration in the former compared to peri-bronchiolar distribution in the latter. Sjogren's syndrome, rheumatoid arthritis, immunodeficiency disorders, and hypersensitivity are conditions associated with follicular bronchiolitis. ${ }^{6}$ Management is usually aimed at treating the underlying condition in the case of follicular bronchiolitis associated with diseases like rheumatoid arthritis. Follicular bronchiolitis observed in HIV disease has been shown to improve with initiation of anti-retroviral drugs. ${ }^{7}$

On the other hand, when we look at bronchiolitis patterns reported in PCD, follicular bronchiolitis has not been reported. The first case of diffuse panbronchiolitis (DPB) occurring in association with PCD in Japan was reported in $1969 .{ }^{2}$ Since then, at least two groups from Japan have reported cases of Kartagener's syndrome with DPB. ${ }^{3-4}$ Mittal et al. from India report a case series with diffuse panbronchiolitis seen amongst patients with Kartagener's syndrome. ${ }^{8}$ This newly found association of follicular bronchiolitis and PCD is important and demands further examination. The reasoning is that follicular bronchiolitis is capable of producing much respiratory distress, cough, and morbidity. ${ }^{10}$ Furthermore, bronchoscopic biopsy is not routine practice for patients with PCD and there is insufficient knowledge about the prevalence of type-specific bronchiolitis other than diffuse bronchiolitis amongst populations with $\mathrm{PCD} .^{3}$ In chronic lung disease, prompt suspicion for the presence of complications, including bronchiolitis and bronchiectasis should lead to appropriate investigation and targeted therapy via bronchodilators, antiinflammatory and antimicrobial drugs. Early intervention in the course of the disease has resulted in better symptom control and long-term outcomes. ${ }^{11}$

There exists evidence behind the use of macrolides in cystic fibrosis bronchiectasis, diffuse bronchiolitis, and other chronic lung diseases. Much of the benefit is from the antiinflammatory and immunomodulatory effects of macrolides. ${ }^{12}$ Our patient has been placed on daily low-dose macrolide therapy over the past 12 months, has noted significant improvement of chronic cough, and has not been readmitted since. Hayakawa et al. report similar benefits with erythromycin in a series of rheumatoid arthritis patients with FB. $^{9}$

\section{Conclusion}

The association of follicular bronchiolitis with PCD has not been reported in the medical literature. The above case may suggest a newer vision towards approach to airway disease, including type-specific bronchiolitis noted in this subset of PCD patients. While follicular bronchiolitis responds to immunosuppressive drugs targeted at the underlying disorder, little is known about FB in PCD.

\section{References}

1. Noone PG, Leigh MW, Sannuti A, Minnix SL, Carson JL, Hazucha M, Zariwala MA, Knowles MR. Primary ciliary dyskinesia: diagnostic and phenotypic features. Am J Respir Crit Care Med. 2004 Feb 15;169(4):45967.

2. Amitani R, Tomioka $H$, Kurasawa $T$, Ishida $T$, Kuze $F$. Clinical and ultrastructural study on primary ciliary dyskinesia. Jpn J Thorac Dis. 1990;28:300-7.

3. Homma S, Kawabata M, Kishi K, Tsuboi E, Narui K, Nakatani T, Saiki S, Nakata K. Bronchiolitis in Kartagener's syndrome. Eur Respir J. 1999 Dec;14(6):1332-9.

4. Horie M, Arai H, Noguchi S, Suzuki M, Sakamoto $Y$, Oka T. Kartagener syndrome with lung cancer and mediastinal tumor. Nihon Kokyuki Gakkai Zasshi. 2010;48:375-8.

5. Travis WD, Galvin JR. Non-neoplastic pulmonary lymphoid lesions. Thorax. 2001 Dec;56(12):964-71.

6. Elicker B, Pereira CA, Webb R, Leslie KO. Highresolution computed tomography patterns of diffuse interstitial lung disease with clinical and pathological correlation. J Bras Pneumol. 2008Sep;34(9):715-44.

7. Shipe R, Lawrence J, Green J, Enfield K. HIVassociated follicular bronchiolitis. Am J Respir Crit Care Med. 2013 Aug 15;188(4):510-1.

8. Mittal V, Shah A. Situs inversus totalis: the association of Kartagener's syndrome with diffuse bronchiolitis and azoospermia. Arch Bronconeumol. 2012 May;48(5):179-82.

9. Hayakawa H, Sato A, Imokawa S, Toyoshima M, Chida K, Iwata M. Bronchiolar disease in rheumatoid arthritis. Am J Respir Crit Care Med 1996;154:153136.

10. Devouassoux G1, Cottin V, Lioté $H$, Marchand $E$, Frachon I, Schuller A, Béjui-Thivolet F, Cordier JF; Groupe d'Etudes et de Recherche sur les Maladies "Orphelines" Pulmonaires (GERM"O"P). Characterisation of severe obliterative bronchiolitis in rheumatoid arthritis. Eur Respir J. 2009 May;33(5):1053-61.

11. Luisi F, Gandolfi TD, Daudt AD, Sanvitto JP, Pitrez PM, Pinto LA. Anti-inflammatory effects of macrolides in childhood lung diseases. J Bras Pneumol. 2012 NovDec;38(6):786-96.

12. Aerni M, Vassallo R, Ryu J. Successful teatment of 
follicular bronchiolitis with macrolide. Chest. 2005; 128

(4_MeetingAbstracts): 428S.

\section{PEER REVIEW}

Not commissioned. Externally peer reviewed.

\section{CONFLICTS OF INTEREST}

The authors declare that they have no competing interests.

\section{PATIENT CONSENT}

The authors, Thalanayar PM and Holguin F declare that:

1. They have obtained written, informed consent for the publication of the details relating to the patient(s) in this report.

2. All possible steps have been taken to safeguard the identity of the patient(s).

3. This submission is compliant with the requirements of local research ethics committees. 